\title{
Natural variants in SARS-CoV-2 S protein pinpoint structural and functional hotspots: implications for prophylaxis and therapeutic strategies
}

Suman Pokhrel ${ }^{1 \dagger}$, Benjamin R. Kraemer ${ }^{1 \dagger}$, Scott Burkholz ${ }^{2}$ and Daria Mochly-Rosen ${ }^{1} *$

${ }^{1}$ Department of Chemical and Systems Biology, Stanford University School of Medicine, Stanford, CA

${ }^{2}$ Flow Pharma, Inc., Pleasant Hill, CA

${ }^{\dagger}$ These authors contributed equally to the study

*Address correspondence to

Daria Mochly-Rosen

Department of Chemical \& Systems Biology, School of Medicine, Stanford University, CA, USA

Email: mochly@stanford.edu

Tel: 650-724-8098 


\begin{abstract}
:
In December 2019, a novel coronavirus, termed severe acute respiratory syndrome coronavirus 2 (SARS-CoV-2), was identified as the cause of pneumonia with severe respiratory distress and outbreaks in Wuhan, China. The rapid and global spread of SARS-CoV-2 resulted in the coronavirus 2019 (COVID-19) pandemic. Earlier during the pandemic, there were limited genetic viral variations. As millions of people became infected, multiple single amino acid substitutions emerged. Many of these substitutions have no consequences. However, some of the new variants show a greater infection rate, more severe disease, and reduced sensitivity to current prophylaxes and treatments. Of particular importance in SARS-CoV-2 transmission are mutations that occur in the Spike $(\mathrm{S})$ protein, the protein on the viral outer envelope that binds to the human angiotensin-converting enzyme receptor (hACE2). Here, we conducted a comprehensive analysis of 441,168 individual virus sequences isolated from humans throughout the world. From the individual sequences, we identified 3,540 unique amino acid substitutions in the S protein. Analysis of these different variants in the $\mathrm{S}$ protein pinpointed important functional and structural sites in the protein. This information may guide the development of effective vaccines and therapeutics to help arrest the spread of the COVID-19 pandemic.
\end{abstract}




\section{Introduction}

To curb the COVID-19 pandemic, most efforts have focused on preventing entry of the virus by inhibiting the interaction of severe acute respiratory syndrome coronavirus 2 (SARSCoV-2) with its human receptor, angiotensin-converting enzyme 2 (hACE2) ${ }^{1}$. Interaction of SARS-CoV-2 with hACE2 occurs via the S protein on the viral envelope. Proteases cleave the S protein into $\mathrm{S} 1$ and $\mathrm{S} 2$ subunits $^{2,3,4}$ to enable viral binding to hACE2 ${ }^{5}$ and viral entry by membrane fusion $^{6}$. The $\mathrm{S}$ protein is a homotrimer and the $\mathrm{S} 1$ subunit of each of the monomers of the S protein contains the receptor-binding domain (RBD; Fig. 1a, b) in either the 'open' (active) or 'closed' (inactive) conformations ${ }^{7,8,9}$ (Supplemental Fig. 1a).

Four main types of prophylaxis or therapeutic strategies, focusing on the $\mathrm{S}$ protein, have been employed: 1). Preventing proteolysis of the $\mathrm{S}$ protein ${ }^{10} ; 2$ ). Competing with $\mathrm{S} 1$ binding to hACE2, using S1 or hACE2 protein fragments or peptides ${ }^{1,11,12}$; 3). Generating monoclonal or polyclonal antibodies against SARS-CoV-2 S protein or RBD, to be used as passive vaccines ${ }^{13}$; and 4) Active vaccines that generate an immune response, usually to the $\mathrm{S} 1$ subunit $^{14,15,16,17}$.

Besides the RBD, the S protein of the coronaviruses, including SARS CoV-2, has several other regions that are predicted to be relatively conserved due to their critical role for $\mathrm{S}$ protein functions. These regions include the trimer interface of $S^{7,9}$, furin proteolysis sites ${ }^{5,6}$, glycosylation sites ${ }^{18,19}$, neuropilin-binding sites $^{20,21,22}$ and linoleic acid (LA)-binding site ${ }^{9,23}$. These regions may be important for maintaining structural integrity, entry, and transmission of the virus and therefore are likely to serve as potential targets for development of prophylaxes and therapeutics.

Although SARS-CoV-2 undergoes mutations at a lower frequency than other viruses like influenza and $\mathrm{HIV}^{24}$, the emergence of several common variants of SARS-CoV-2 in human populations may generate resistance to current prophylaxis and therapeutics. Some of these 
mutations result in gain of fitness for the virus due to mutations in the $\mathrm{S}$ protein ${ }^{25,26,27,28}$. Early in the pandemic, in February 2020, a single missense mutation resulting in a change from aspartate to glycine in position 614 (D614G) emerged in Europe and became the dominant variant of the virus. The D614G variant has spread throughout the world and increased the transmissibility of SARS-CoV-2 by conferring higher viral loads in young hosts without an apparent increase in the severity of the disease ${ }^{29}$. With the emergence of new variants, such as B.1.1.7 (also known as the UK variant) and B.1.351 (also known as the South African variant) that have greater transmissibility and escape antibody detection ${ }^{25,26,27,28}$ (Table 1), it is imperative to map other substitutions in the S protein sequence. Such substitutions may contribute to future variants that lead to increased transmissibility or to variants that evade prophylaxis or therapeutics. Particularly, amino acid substitutions in the RBD, including those that interact directly with hACE2 $2^{25,26,27,28}$ (Fig. 1c) may have an impact. Here, we aimed to identify regions on the $\mathrm{S}$ protein that are relatively invariant to guide prophylaxis and therapeutic development more efficiently.

\section{Results}

\section{SARS-CoV-2 Spike Protein}

The SARS-CoV-2 S protein is 1273 amino acids long; it contains a signal peptide (amino acids 1-13), the S1 subunit (14-685 residues) that mediates receptor binding, and the S2 subunit (686-1273 residues) that mediates membrane fusion ${ }^{30}$. To identify areas in the $\mathrm{S}$ protein that are the least divergent as the virus evolves in humans, we obtained viral sequences from GISAID (Supplemental Table 1) that as of March 1, 2021, included 633,137 individual virus sequences isolated from humans throughout the world. As compared with the index WIV04 (MN996528.1, also known as the Wuhan variant or index virus) sequence of February $2020^{31}$, the 1,273 amino 
acid $\mathrm{S}$ protein ${ }^{8}$ had 3,540 variants. This number of variants only includes filtered sequences $(441,168)$ that are complete and do not contain an abnormal number of mutations (see Methods). As there are 3,540 variants, on average, each position in the 1273 amino acid protein sequence has approximately three variants (Fig. 1d). However, some regions harbor 9 variants in a single amino acid position whereas others have no variants (Fig. 1d; Supplemental Table 3). Regions in S protein with 2 or fewer variants/position (marked in light blue, Fig. 1d, e) are more prevalent in the structurally critical trimer interface (46\% of the amino acids; Fig. 1d, Supplementary Fig. 1b, c, see Supplementary Table 4), and in the RBD (56\%, Fig. 1e, Supplementary Fig. 1b, c). There are a total of 123 positions that are entirely invariable (Supplementary Table 3).

\section{Receptor Binding Domain}

Much of the prophylaxis and therapeutic efforts are focused on the RBD (amino acids 331-524). Among the 3,540 variant sequences, we found only 22 invariant amino acids in the RBD (Fig. 1e, marked by dots under the position; Supplemental Table 3). Of those amino acid substitutions in the RBD, only $3 \%$ are predicted by PROVEAN software ${ }^{32}$ to be structurally or functionally damaging (Supplemental Table 2). Using PROVEAN, we also examined the predicted impact of the amino acid substitutions in the common more infective variants (B.1, B.1.1.7, B.1.351, B.1.427/429, B.1.256 and P.1; Table1) on the RBD structure and function and found that these variants are predicted to have a neutral effect, suggesting these variants are not decreasing the fitness of the virus.

\section{Furin Proteolysis Sites}

We next examined other regions in the $\mathrm{S}$ protein for which functions have been assigned. Furin proteolysis at the S1-S2 boundary (681-685) and in S2 (811-815) exposes the RBD to enable hACE2 binding, and the S2 domain to initiate membrane fusion ${ }^{5}$. Recent studies show that these cleavage sites are not necessarily specific for furin-mediated proteolysis and that $\mathrm{S}$ 
may be processed by multiple proteases to open the RBD into the active conformation ${ }^{2,3,4,33}$. Consistent with these observations, both the furin proteolysis consensus sites and the arginine that are critical for proteolysis are not conserved in the S protein (Fig. 2a), in agreement with a prior analysis of furin site $1^{34}$.

\section{Glycosylation Sites}

The S protein also has 66 glycosylation sites in each trimer, which facilitate protein folding and may lead to host immune system evasion ${ }^{19}$, as $40 \%$ of the S protein's surface is shielded by glycans ${ }^{18}$. Surprisingly, with one exception, none of these glycosylation sites were invariable, suggesting that not all the glycosylation sites are essential for the S protein's functions (Fig. 2b,c). The only asparagine serving as an invariable glycosylation site is N343 in the RBD domain, located more than $25 \AA$ away from hACE2-binding site, and therefore unlikely to mediate receptor binding.

\section{Neuropilin-1 Interaction Site}

Neuropilin-1 (NRP-1) is a transmembrane receptor that regulates angiogenesis ${ }^{35}$ and immune response ${ }^{36}$ and is expressed in many cell types ${ }^{36}$ such as the endothelium ${ }^{37}$, immune cells $^{38}$, and neurons ${ }^{39}$. Interaction between NRP-1 and S protein was proposed to regulate SARSCoV-2 transmission ${ }^{20,21,22}$. Proteolysis of $\mathrm{S} 1$ index variant by furin was found to expose a C terminal motif, RXXR (where $\mathrm{R}$ is arginine and $\mathrm{X}$ is any amino acid), known to be the binding motif in NRP-1 $1^{20,22}$. For example, a monoclonal antibody against the RXXR-binding site on NRP-1 SARS-CoV-2 reduced infectivity in culture ${ }^{22}$. Nevertheless, we found that the NRP-1 interaction-site in S1 is not conserved (Fig. 2d). Although the variants are predicted to have a neutral effect on the S1 protein structure (using PROVEAN analysis, Supplemental Table 2), $90 \%$ of the positions in the NRP1-interaction site have more than 2 variants (or an average of 4.3 variants/position; Fig. 2d). 


\section{Linoleic Acid-Binding Site}

A fatty acid-binding pocket has been identified in the inactive conformation of $\mathrm{S}$ protein ${ }^{9}$ (Fig. 3a, b). The amino acids that make this pocket are conserved in other coronaviruses ${ }^{9}$ and are unchanged (less than 2 variants) in $75 \%$ of the position (Fig. 3a, b). Furthermore, among the 20 amino acids that line this pocket, $71 \%$ of the identified variants are predicted to have a neutral effect using PROVEAN (Supplemental Table 2). Analysis of the LA-bonding site identifies a potential pharmacophore that may fit small molecules (Fig. 3c), perhaps by mimicking $\omega-3$ fatty $\operatorname{acids}^{23}$.

\section{Relatively Invariable Regions with Unidentified Function}

We also identified another less variable region between residues 541-612 (Fig. 4); 62\% of the amino acid positions in this region have 2 or fewer variants and 12 positions are entirely invariable ('Hot Region'; Fig. 1a and Fig. 4a, b). This less variable region is relatively hydrophobic, yet a substantial number of residues remain exposed in the open and closed conformations (Fig. 4c). Six residues, V551, T553, C590, V595, V608, Y612, in this relatively invariable region form a part of the largest hydrophobic patch in the protein measuring $370 \AA^{2}$ (Fig. 4d, e). Five of these residues (excluding T553) along with other residues that make this hydrophobic patch tolerate very few mutations and almost all the mutations that are tolerated change to hydrophobic amino acids (Fig. 4d). We examined this region using Site Finder in $\mathrm{MOE}^{40}$ and found that there is a binding site with a positive score for the propensity of ligand binding ${ }^{41}$, which encompasses several residues from this region (i.e. Cys590, Ser591, Phe592, Gly593) (Supplemental Fig. 1e). This hydrophobic region is also 81\% identical between SARS$\mathrm{CoV}$ and SARS-CoV-2, but less than $15 \%$ identical when comparing the SARS-CoV-2 sequence with that of MERS-CoV (Fig. 4f).

\section{Discussion}


While SARS-CoV-2 has a lower mutation rate than other viruses due to proof-reading mechanisms ${ }^{24}$, aspects such as a relatively high $\mathrm{R}_{0}$ of 1.9 to $2.6^{42}$, comparatively long asymptomatic incubation and infection periods, and zoonotic origins, leads to high variability in mutations in specific regions compared to the original reference sequence. This has been illustrated with the divergence of 6 major lineages in the past few months (Table 1). Our analysis of the frequency of variants throughout the S protein of SARS-CoV-2 identified regions of high and low divergence, which may aid in developing effective prophylactic and therapeutic treatments. In this analysis of mutations in the $\mathrm{S}$ protein, we did not consider the frequency of a particular mutation or in how many countries the mutation was found. Such analysis, as was done for $\mathrm{D} 614 \mathrm{G}^{43}$, may further aid in determining the potential improved viral fitness acquired by a particular mutation.

Protein glycosylation is essential for viral infection. ${ }^{44}$ In SARS-CoV-2 S protein, there are 22 known $\mathrm{N}$-glycosylation sites per monomer (Fig. 2b, c), but only one, asparagine 343, appears to be conserved. Furthermore, we found 156 positions in $\mathrm{S}$ that mutate to an asparagine residue in the existing 3,540 variants that we analyzed, and many of them are exposed on the S protein (Supplemental Fig. 1d). We propose that some of these new asparagine residues may create new glycosylation sites on the $\mathrm{S}$ protein that can contribute to immune evasion. Such an impact on the immune evasion by changes in the positions of glycosylation sites of viral envelope proteins have been described for influenza viruses; e.g., H3N2 has numerous new Nlinked glycans on the viral hemagglutinin that enabled the virus to escape antibody neutralization and evade the host's immune system ${ }^{45}$. The formation of new glycosylation positions may also affect viral susceptibility to existing antibodies and to the immune response of infected individuals. A cryo-electron microscopy study has already suggested that coronaviruses mask important immunogenic sites on their surface by glycosylation ${ }^{46}$. Furthermore, recent work 
suggests that changes in glycosylation sites may affect its recognition by other potential human proteins and receptors, inducing the toll-like receptors, calcitonin-like receptors, and heat shock protein GRP78, thus leading to a more severe inflammation that characterizes a more severe form of COVID-19

Additional sites on the $\mathrm{S}$ protein have been suggested to be critical for viral infectivity, including the trimer interface, the furin proteolysis sites and the NRP-1 binding site. However, our analysis suggests that not all these sites will be effective targets for prophylaxis and therapeutics. Specifically, the trimer interface is less accessible and therefore unlikely to be druggable. Another issue relates to the furin sites. As the viral $\mathrm{S}$ protein activation appears to require furin proteolysis ${ }^{2,3,4}$, protease-specific inhibitors are tested as a means to protect from infection ${ }^{48}$. However, our analysis suggests that this may not be an effective strategy, given the high variability of furin cleavage sites. This suggestion is consistent with previous data showing that other proteinases expressed throughout the body may work synergistically to activate the $\mathrm{S}$ protein $^{2,33}$. Therefore, drugs that focus on inhibiting any single protease may not be effective preventative treatment against all SARS-CoV-2 variants. Similarly, the NRP1-binding site that is generated by proteolysis and the exposure of a C-terminal RXXR motif ${ }^{20,22}$ may not be a good target for treatment against all SARS-CoV-2 variants, unless such a motif is created by other proteases.

Are there additional sites on the $\mathrm{S} 1$ protein that can be explored to identify new treatments of COVID-19 or prevention of infections by SARS-CoV-2? There might be a benefit in focusing on the LA-binding site that help stabilize the $\mathrm{S}$ in the inactive closed conformer. Small molecules that mimic LA and bind into the LA pocket may stabilize the S protein in the closed/inactive conformation, thus reducing infectivity (Fig. 3). Therefore, exploring the LA pharmacophore (Fig. 3c) with small molecules that can hold the S-protein in closed 
conformation, thus preventing the presentation of RBD to hACE2, could be of great interest as this may reduce viral infectivity. Our data also suggest that it may be beneficial to develop passive and active vaccines that target the RBD, instead of the entire glycosylated $\mathrm{S}$ protein; the $\mathrm{RBD}$ is less variable relative to the whole $\mathrm{S} 1$ protein (compare Fig. 1e to 1d). However, similar to some of the common viral isolates, such as the South African, B.1.351, new amino acid substitutions in the RBD may evade such therapeutics; e.g., loss of immunoreactivity to monoclonal antibodies ${ }^{25}$.

Finally, our study suggests that drugs and antibodies targeting region 541-612, a relatively conserved and exposed region on the protein's surface that we identified (Fig. 4), warrant further study. Determining how druggable the pocket encompassing this region is (residues Cys590, Ser591, Phe592, Gly593), provided its solvent exposure, and whether modulating $\mathrm{S}$ protein by engaging this site will have a biological consequence is a challenge (Supplemental Fig. 1e). Very recently, Q564 within this region (star in Fig. 4a) has been proposed to act as a 'latch', stabilizing the closed/inactive conformation of the $\mathrm{S}$ protein ${ }^{49}$. The high degree of conservation of hydrophobicity in this region potentially indicates its role in membrane fusion and/or maintaining structural integrity. The sequence similarity between SARS-CoV-2 and SARS-CoV (Fig. 4f) further supports the importance of this region, especially as both viruses have a similar route of infection. Determining the role of this invariable region warrant a further study, as it may be another Achilles heel to target for anti-SARS-CoV-2 treatment. 


\section{Materials \& Methods}

Database of $S$ protein amino acid variants, the world regions from where the virus was obtained, and whether the sequence is predicted to be deleterious.

A FASTA formatted file containing 633,137 spike protein sequences was retrieved on 03/01 from the GISAID database. This file had previously been preprocessed by the database with the individual alignment of genomes to the WIV04 (MN996528.1 $1^{31}$ ) reference sequence, using mafft [https://doi.org/10.1093/molbev/mst010], via the command "mafft --thread 1 --quiet input.fasta > output.fasta" with subsequent translation into protein from the spike protein-coding region at 21563 to 25384.

For the analysis in this paper, only sequences sampled from humans were retrieved with the spike protein sequences realigned via mafft [https://doi.org/10.1093/molbev/mst010] against the WIV04 (MN996528.1, http://dx.doi.org/10.1038/s41586-020-2012-7) reference utilizing parameters ideal for a large number of highly similar protein sequences as well as using the option to maintain position numbering against the reference.

"grep -i "|Human|" input.fasta -A1 > output.fasta"

"mafft --6merpair --thread -1 --keeplength --addfragments input.fasta reference.fasta > output.fasta"

A python script (supplementary material) was generated to filter sequences based on set quality thresholds that included 1) 0 ambiguous protein positions; 2) 0 deletions or gaps outside of common deletions including position 69,70 and 144/145; 3) only full length pre-alignment of 1273 but down to 1270 in the event of the specified deletions; and 4) a maximum of less than $1 \%$ (13) amino acid substitutions from reference. These resulting 441,168 sequences (supplementary table), were chosen by the strict quality thresholds to remove low quality and potentially error 
prone sequences based on those that were incomplete, contain uncommon deletions, insertions, and have an unusually high number of mutations.

\section{Calculating Number of Variants}

The raw data for variants in the $\mathrm{S}$ protein (see Extended Material Table) was read into R studio (v. 1.3.1093) and analyzed using the Tidyverse package. The number of unique variants was calculated for each position, excluding insertions. Graphs were created for specific regions and each position was color-coded according to the number of variants present in that position (i.e., 0 - no color, 1-2 is blue, 3-4 is yellow, $>5$ is red). See sample code below:

Calculating variants:

$\mathrm{df} \%>\%$

group_by(Position, .drop=FALSE)\%>\%

tally()

Graphing Example:

ggplot(df)+ \#graph of RBD, works for diff colors

$$
\begin{aligned}
& \text { geom_col(data }=\operatorname{subset}(\mathrm{df}, \text { Position }>330 \& \text { Position }<525) \text {, aes }(\mathrm{x}=\text { Position, } \mathrm{y}=(\mathrm{n}) \text {, } \\
& \text { fill=as.factor(n)))+ } \\
& \text { ggtitle("RBD") + } \\
& \text { scale_fill_manual }(\text { values = pal, name = "Number" })+ \\
& \operatorname{labs}(\mathrm{y}=\text { "Number of Mutations")+ } \\
& \text { theme }(\text { panel.background = element_blank }(), \text { text=element_text }(\operatorname{size}=20))
\end{aligned}
$$

For the functional regions, the proportion of positions with 2 or fewer observed variants was calculated. See formula below:

Proportion with regions with 2 or fewer $=\frac{\# \text { of Positions with } 2 \text { or fewer variants }}{\text { Total } \# \text { of Positions }} * 100 \%$ 


\section{Calculating Predicted Effect of Variants in PROVEAN}

The amino acid sequence of S protein from the reference EPI_ISL_402124 (WIV04; Wuhan) sequence was uploaded to PROVEAN (http://provean.jcvi.org/index.php). Every variant observed in $\mathrm{S}$ was also uploaded to compare against the reference sequence. Each variant was either predicted to be 'deleterious' or 'neutral'. The PROVEAN predictions were also read into R studio (v. 1.3.1093) and analyzed with the Tidyverse package for every region analyzed. The proportion of variants predicted to be neutral and deleterious were calculated for the functional regions analyzed in S. See Supplemental Table 1. Sample code below:

Calculating PROVEAN ratios:

table(df\$ProveanPrediction)\%>\%

prop.table()\%>\%

$\operatorname{round}(4)$

\section{Protein Structures}

Molecular Operating Environment (MOE) software $^{40}$ was used to prepare the figures using PDB ID: $7 \mathrm{~A} 98^{4}$ for Figures 1b-d, 2d; Supplemental Fig. 1a (left), d (left), Supplemental Fig. 2a, and PDB ID: $6 \mathrm{ZB}^{6}{ }^{6}$ was used to prepare Fig. 2a, Supplemental Fig. 1a (right), d (right).

\section{Sequence Alignment}

The Spike protein sequences from SARS-CoV-2, SARS-CoV, and MERS-CoV were uploaded to Jalview $^{50}$. The Mafft alignment was then performed to align each amino acid sequence.

\section{Pharmacophore Generation}

PDB ID: $6 \mathrm{ZB5}^{6}$ was opened and prepared using the QuickPrep functionality at the default settings in MOE as mentioned previously. Dummy atoms were created at the LA-binding site formed by chains 6ZB5.A and 6ZB5.C. AutoPH4 tool ${ }^{51,52}$ was used to generate the pharmacophore at the dummy atom site in the Apo generation mode. 


\section{Acknowledgments}

Supported in part by the 2020 COVID-19 Response: Drug and Vaccine Prototyping Grant from the Innovative Medicines Accelerator, Stanford University to D. M.-R. We gratefully thank the many investigators throughout the world that provided the SARS-CoV-2 sequences to this public database.

\section{Author Contributions}

S.P., B.R.K., S.B. provided data analysis, visualization, and draft writing. D. M.-R. conceived the project, supervised the analysis and writing.

\section{Competing Interests Statements}

The authors declare no competing interests. 


\section{References}

1. Yang, J. et al. Molecular interaction and inhibition of SARS-CoV-2 binding to the ACE2 receptor. Nat. Commun. 11, 4541 (2020).

2. Fuentes-Prior, P. Priming of SARS-CoV-2 S protein by several membrane-bound serine proteinases could explain enhanced viral infectivity and systemic COVID-19 infection. $J$. Biol. Chem 296, 100135-100136 (2021).

3. Belouzard, S., Chu, V. C. \& Whittaker, G. R. Activation of the SARS coronavirus spike protein via sequential proteolytic cleavage at two distinct sites. PNAS 106, 5871-5876 (2009).

4. Tang, T. et al. Proteolytic Activation of SARS-CoV-2 Spike at the S1/S2 Boundary: Potential Role of Proteases beyond Furin. ACS Infect. Dis. 12, 264-272 (2021).

5. Hoffmann, M. et al. SARS-CoV-2 cell entry depends on ACE2 and TMPRSS2 and is blocked by a clinically proven protease inhibitor. Cell 181, 271-280.e8 (2020).

6. Xia, S. et al. Fusion mechanism of 2019-nCoV and fusion inhibitors targeting HR1 domain in spike protein. Cell. Mol. Immunol. 17, 765-767 (2020).

7. Benton, D. J. et al. Receptor binding and priming of the spike protein of SARS-CoV-2 for membrane fusion. Nature (2020) doi:10.1038/s41586-020-2772-0.

8. Wrapp, D. et al. Cryo-EM structure of the 2019-nCoV spike in the prefusion conformation. Science. 367, 1260-1263 (2019).

9. Toelzer, C. et al. Free fatty acid binding pocket in the locked structure of SARS-CoV-2 spike protein Downloaded from. Science. 370, 725-730 (2020).

10. Cheng, Y. W. et al. Furin inhibitors block SARS-CoV-2 spike protein cleavage to suppress virus production and cytopathic effects. Cell Rep. 33, (2020).

11. Larue, R. C. et al. Rationally Designed ACE2-Derived Peptides Inhibit SARS-CoV-2. 
Bioconjug. Chem. 32, 215-223 (2020).

12. de Vries, R. D. et al. Intranasal fusion inhibitory lipopeptide prevents direct-contact SARS-CoV-2 transmission in ferrets. Science. eabf4896 (2021) doi:10.1126/science.abf4896.

13. Liu, L. et al. Potent neutralizing antibodies against multiple epitopes on SARS-CoV-2 spike. Nature 584, 450-456 (2020).

14. Jackson, L. A. et al. An mRNA vaccine against SARS-CoV-2 - preliminary report. $N$. Engl. J. Med. 383, (2020).

15. Mulligan, M. J. et al. Phase I/II study of COVID-19 RNA vaccine BNT162b1 in adults. Nature 586, 589-593 (2020).

16. Walsh, E. E. et al. Safety and immunogenicity of two RNA-based Covid-19 vaccine candidates. N. Engl. J. Med. (2020) doi:10.1056/nejmoa2027906.

17. West, A. P., Barnes, C. O., Yang, Z. \& Bjorkman, P. J. SARS-CoV-2 lineage B.1.526 emerging in the New York region detected by software utility created to query the spike mutational landscape. BioRxiv (2021) doi:10.1101/2021.02.14.431043.

18. Grant, O. C., Montgomery, D., Ito, K. \& Woods, R. J. Analysis of the SARS-CoV-2 spike protein glycan shield reveals implications for immune recognition. Sci. Rep. 10, 14991 (2020).

19. Watanabe, Y. et al. Vulnerabilities in coronavirus glycan shields despite extensive glycosylation. Nat. Commun. 11, 2688, (2020).

20. Cantuti-Castelvetri, L. et al. Neuropilin-1 facilitates SARS-CoV-2 cell entry and infectivity. Science. 370, 856-860 (2020).

21. Mayi, B. S. et al. The role of Neuropilin-1 in COVID-19. PLoS Pathog. 17, (2021).

22. Daly, J. L. et al. Neuropilin-1 is a host factor for SARS-CoV-2 infection. Science. 370, 
861-865 (2020).

23. Goc, A., Niedzwiecki, A. \& Rath, M. Polyunsaturated $\omega-3$ fatty acids inhibit ACE2-

controlled SARS-CoV-2 binding and cellular entry. Sci. Rep. 11, 5207 (2021).

24. Callaway, E. Makkng sense of coronavirus mutations. Nature 585, 174-177 (2020).

25. Thomson, E. C. et al. Circulating SARS-CoV-2 spike N439K variants maintain fitness while evading antibody-mediated immunity. Cell (2021) doi:10.1016/j.cell.2021.01.037.

26. Yurkovetskiy, L. et al. Structural and Functional Analysis of the D614G SARS-CoV-2 Spike Protein Variant. Cell (2020) doi:10.1016/j.cell.2020.09.032.

27. Davies, N. G. et al. Estimated transmissibility and impact of SARS-CoV-2 lineage B.1.1.7 in England. Science (2021) doi:10.1126/science.abg3055.

28. Tegally, H. et al. Emergence of a SARS-CoV-2 variant of concern with mutations in spike glycoprotein. Nature (2021) doi:10.1038/s41586-021-03402-9.

29. Volz, E. et al. Evaluating the Effects of SARS-CoV-2 Spike Mutation D614G on Transmissibility and Pathogenicity. Cell 184, 64-75.e11 (2021).

30. Huang, Y., Yang, C., Xu, X., Xu, W. \& Liu, S. Structural and functional properties of SARS-CoV-2 spike protein: potential antivirus drug development for COVID-19. Acta Pharmacol. Sin. 41, 1141-1149 (2020).

31. Zhou, P. et al. A pneumonia outbreak associated with a new coronavirus of probable bat origin. Nature 579, 270-273 (2020).

32. Choi, Y. \& Chan, A. P. PROVEAN web server: A tool to predict the functional effect of amino acid substitutions and indels. Bioinformatics 31, 2745-2747 (2015).

33. Seyran, M. et al. The structural basis of accelerated host cell entry by SARS-CoV-2. FEBS J. (2020) doi:10.1111/febs.15651.

34. Xing, Y., Li, X., Gao, X. \& Dong, Q. Natural polymorphisms are present in the furin 
cleavage site of the SARS-CoV-2 spike glycoprotein. Front. Genet. 11, (2020).

35. Kong, J. S. et al. Anti-neuropilin-1 peptide inhibition of synoviocyte survival, angiogenesis, and experimental arthritis. Arthritis Rheum. 62, 179-190 (2010).

36. Guo, H. F. \& Vander Kooi, C. W. Neuropilin functions as an essential cell surface receptor. J. Biol. Chem. 290, 29120-29126 (2015).

37. Soker, S., Takashima, S., Miao, H. Q., Neufeld, G. \& Klagsbrug, M. Neuropilin-1 Is Expressed by Endothelial and Tumor Cells as an Isoform-Specific Receptor for Vascular Endothelial Growth Factor. Cell 92, 735-745 (1998).

38. Schellenburg, S., Schulz, A., Poitz, D. M. \& Muders, M. H. Role of neuropilin-2 in the immune system. Mol. Immunol. 90, 239-244 (2017).

39. Erskine, L. et al. VEGF-A and neuropilin 1 (NRP1) shape axon projections in the developing CNS via dual roles in neurons and blood vessels. Dev. 144, 2504-2516 (2017).

40. Chemical Computing Group ULC. Molecular Operating Environment (MOE). (2021).

41. Soga, S., Shirai, H., Koborv, M. \& Hirayama, N. Use of amino acid composition to predict ligand-binding sites. J. Chem. Inf. Model. 47, 400-406 (2007).

42. Locatelli, I., Trächsel, B. \& Rousson, V. Estimating the basic reproduction number for COVID-19 in Western Europe. PLoS One 16, e0248731 (2021).

43. Fang, S. et al. GESS: a database of global evaluation of SARS-CoV-2/hCoV-19 sequences. Nucleic Acids Res. (2020) doi:10.1093/nar/gkaa808.

44. Watanabe, Y., Bowden, T. A., Wilson, I. A. \& Crispin, M. Exploitation of glycosylation in enveloped virus pathobiology. Biochimica et Biophysica Acta - General Subjects vol. 1863 1480-1497 (2019).

45. Allen, J. D. \& Ross, T. M. H3N2 influenza viruses in humans: Viral mechanisms, evolution, and evaluation. Hum. Vaccin. Immunother. 14, 1840-1847 (2018). 
46. Walls, A. C. et al. Glycan shield and epitope masking of a coronavirus spike protein observed by cryo-electron microscopy. Nat. Publ. Gr. 23, 899-907 (2016).

47. Gadanec, L. K. et al. Molecular Sciences Can SARS-CoV-2 Virus Use Multiple Receptors to Enter Host Cells? Int. J. Mol. Sci. 22, 992-1328 (2021).

48. Papa, G. et al. Furin cleavage of SARS-CoV-2 Spike promotes but is not essential for infection and cell-cell fusion. PLoS Pathog. 17, (2021).

49. Peters, M. H., Bastidas, O., Kokron, D. S. \& Henze, C. E. Static all-atom energetic mappings of the SARS-Cov-2 spike protein and dynamic stability analysis of 'Up' versus ‘Down' protomer states. PLoS One 15, e0241168 (2020).

50. Waterhouse, A. M., Procter, J. B., Martin, D. M. A., Clamp, M. \& Barton, G. J. Jalview Version 2-A multiple sequence alignment editor and analysis workbench. Bioinformatics 25, 1189-1191 (2009).

51. Jiang, S., Feher, M., Williams, C., Cole, B. \& Shaw, D. E. Autoph4: An automated method for generating pharmacophore models from protein binding pockets. J. Chem. Inf. Model. 60, 4326-4338 (2020).

52. Chemical Computing Group ULC. AutoPH4, Scientific Vector Language (SVL). (2021). 
Table 1. Common variants of concern.

\begin{tabular}{|c|c|c|c|}
\hline Variant & Geographic Location & $\begin{array}{c}\text { Relevant Amino Acid } \\
\text { Substitutions }\end{array}$ & $\begin{array}{l}\text { PROVEAN } \\
\text { Prediction }\end{array}$ \\
\hline B.1 Lineage & Europe & D614G & Neutral \\
\hline \multirow[b]{4}{*}{ B1.1.7 } & \multirow[b]{4}{*}{ UK } & N501Y & Neutral \\
\hline & & A570D & Neutral \\
\hline & & D614G & Neutral \\
\hline & & $\mathrm{P} 681 \mathrm{H}$ & Neutral \\
\hline \multirow[b]{4}{*}{ B.1.351 } & \multirow[b]{4}{*}{ South Africa } & $\mathrm{K} 417 \mathrm{~N}$ & Neutral \\
\hline & & E484K & Neutral \\
\hline & & N501Y & Neutral \\
\hline & & D614G & Neutral \\
\hline B.1.427/B.1.429 & California & L452R & Neutral \\
\hline \multirow[b]{4}{*}{ B.1.526 } & \multirow{4}{*}{$\begin{array}{c}\text { Northeastern USA, } \\
\text { New York }\end{array}$} & E484K & Neutral \\
\hline & & S477N & Neutral \\
\hline & & D614G & Neutral \\
\hline & & A701V & Neutral \\
\hline \multirow[b]{5}{*}{ P.1 } & \multirow[b]{5}{*}{ Brazil } & E484K & Neutral \\
\hline & & $\mathrm{K} 417 \mathrm{~N}$ & Neutral \\
\hline & & K417T & Neutral \\
\hline & & N501Y & Neutral \\
\hline & & D614G & Neutral \\
\hline
\end{tabular}




\section{Natural variants in SARS-CoV-2 Figures}

Figure 1. Functional regions in $\mathrm{S}$ protein and the RBD-hACE2 interaction site. a) S protein homotrimer with ribbons colored according to legend, bound to hACE2 (red). Black dotted outline shown in b. b) RBDhACE2 interface. c) RBD-hACE2 interface highlighting residues in RBD within $4.5 \AA$ from hACE2. d) The number of variants per position across the entire sequence of $\mathrm{S}$ protein, highlighting specific functional regions. e) The number of variants per position across RBD. Black dots indicate invariable positions.

a

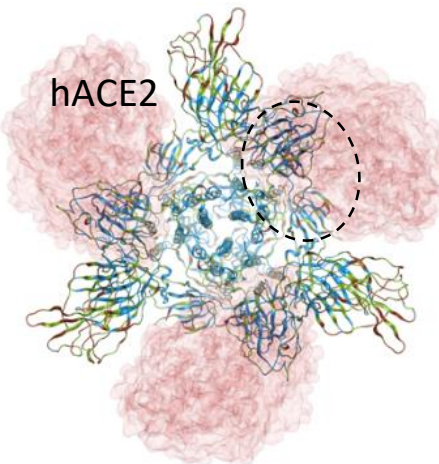

b

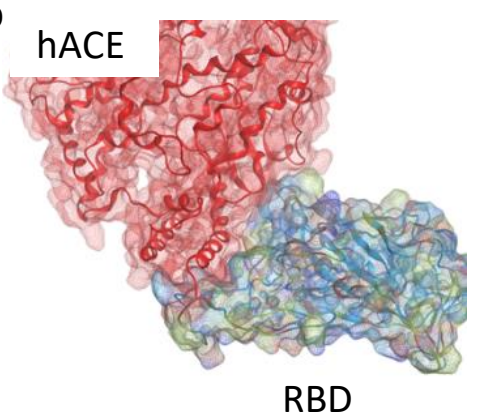

C

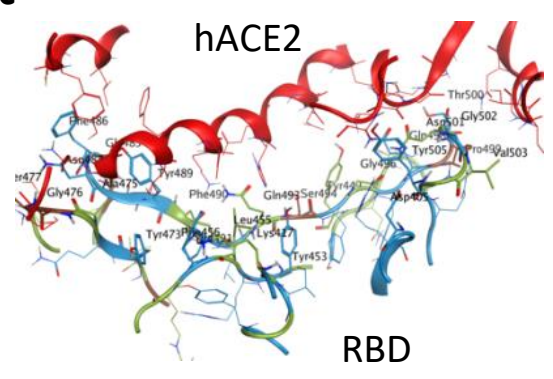

d Spike Protein

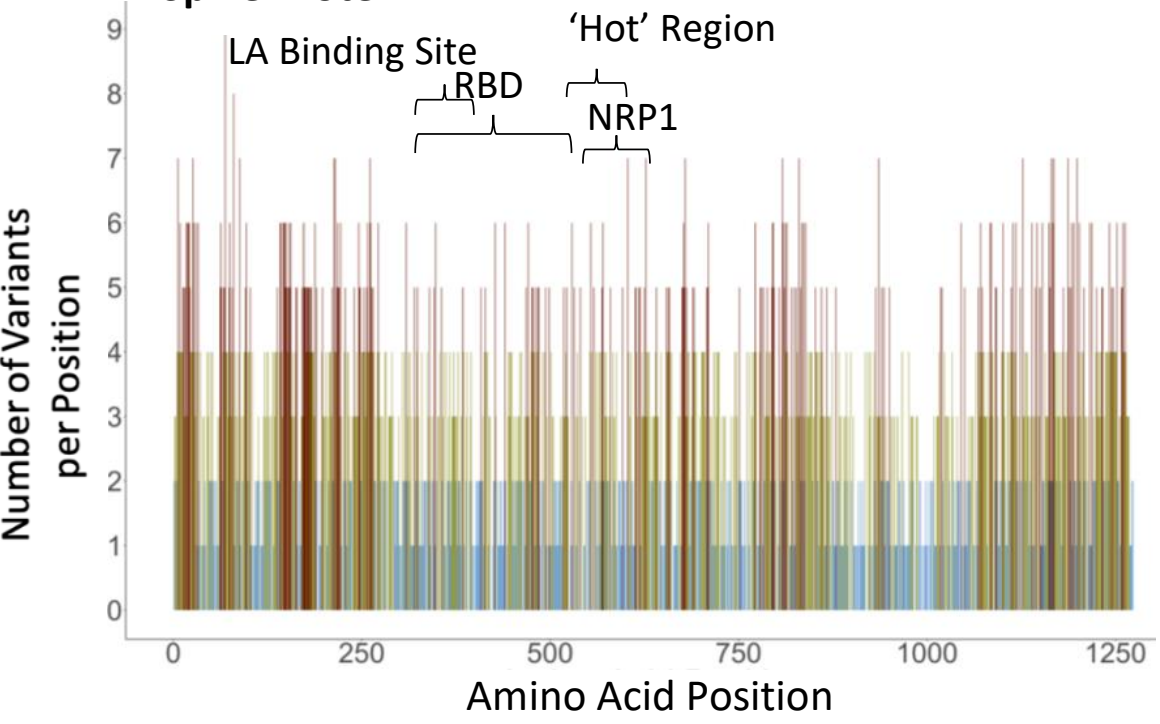

Number of Variants per Position

e

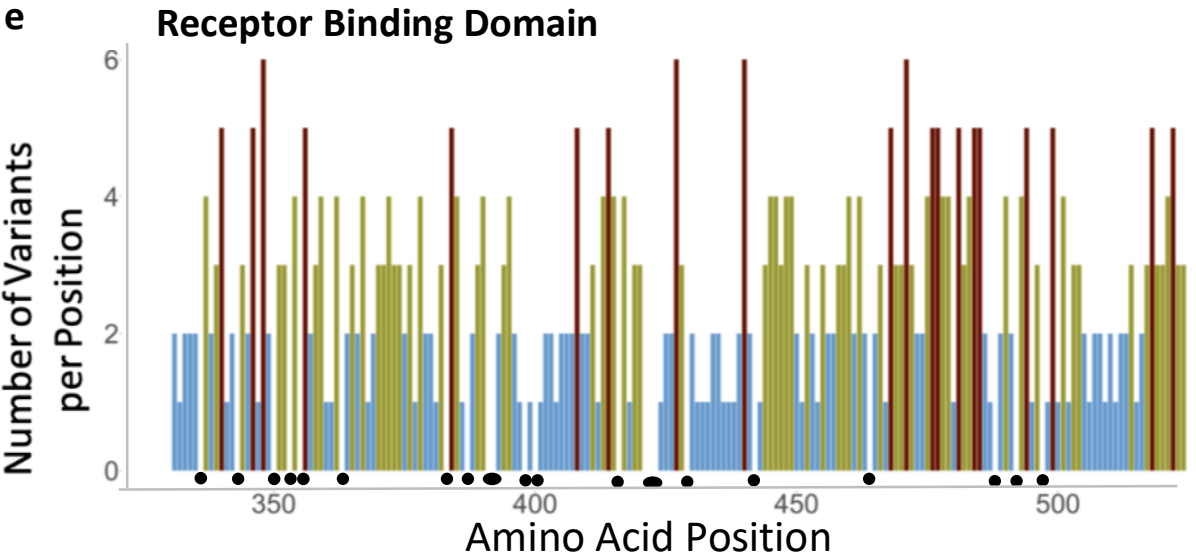


Figure 2. Furin cleavage sites, glycosylation sites, and NRP-1 interaction site. a) Furin cleavage sites in the $\mathrm{S}$ protein. Amino acid indicated below each bar indicate the sequence in the WIV04 index isolate. Variants in other SARS-CoV-2 isolates are indicated within the bars of the graphs using one letter abbreviations for the amino acids. b) The 22 glycosylation sites in the S protein; indicated are the number of variants per position. c) Glycosylation asparagine sites (numbered) are highlighted in pink in the $S$ protein. D) The number of variants in the proposed NRP1-binding site.

a

Furin Site 1

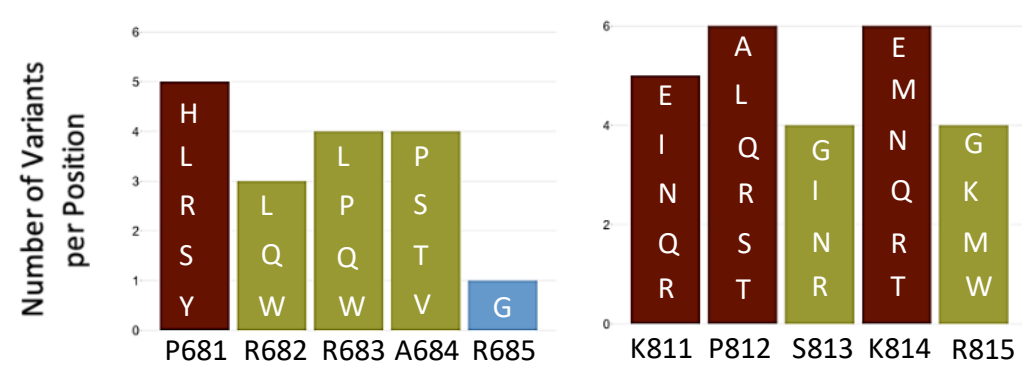

Furin Site 2 b

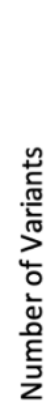

Glycosylation Sites

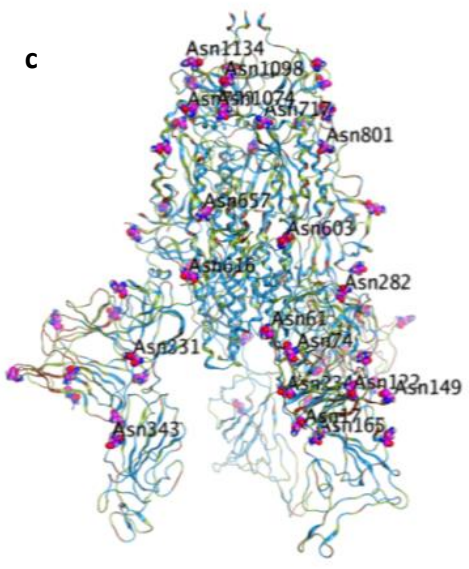

d NRP1 Binding Site

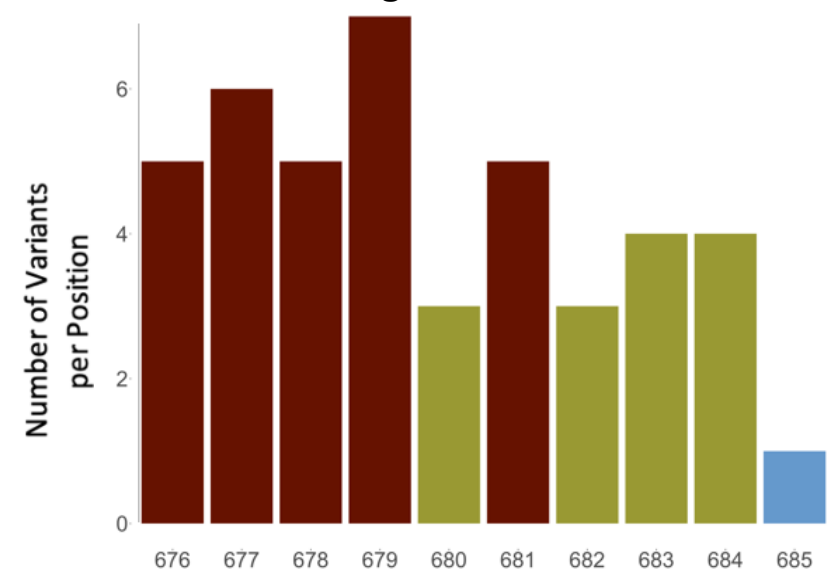

Amino Acid Position 
Figure 3. The LA-binding site in the S protein. a) Hydrophobic pocket forms the LA-binding site. b) The number of variants per position across the LA-binding site; black outlines indicate the positions that form the LA-pocket. c) Pharmacophore of the LA-binding pocket.

a

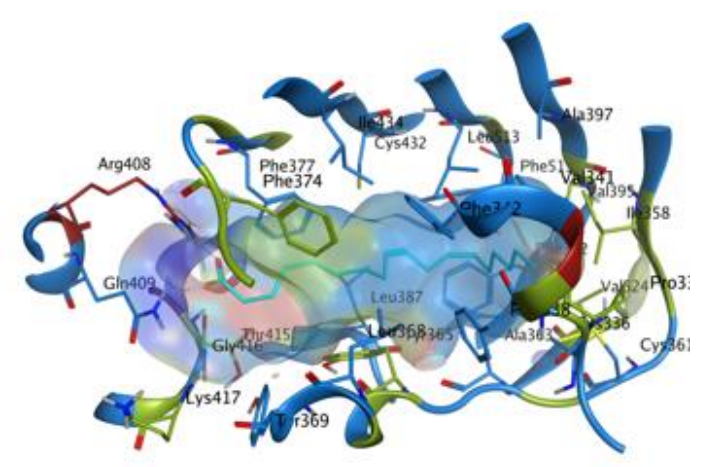

b

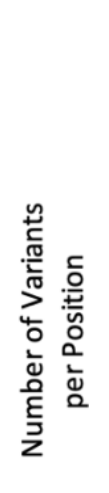

LA-Binding Site

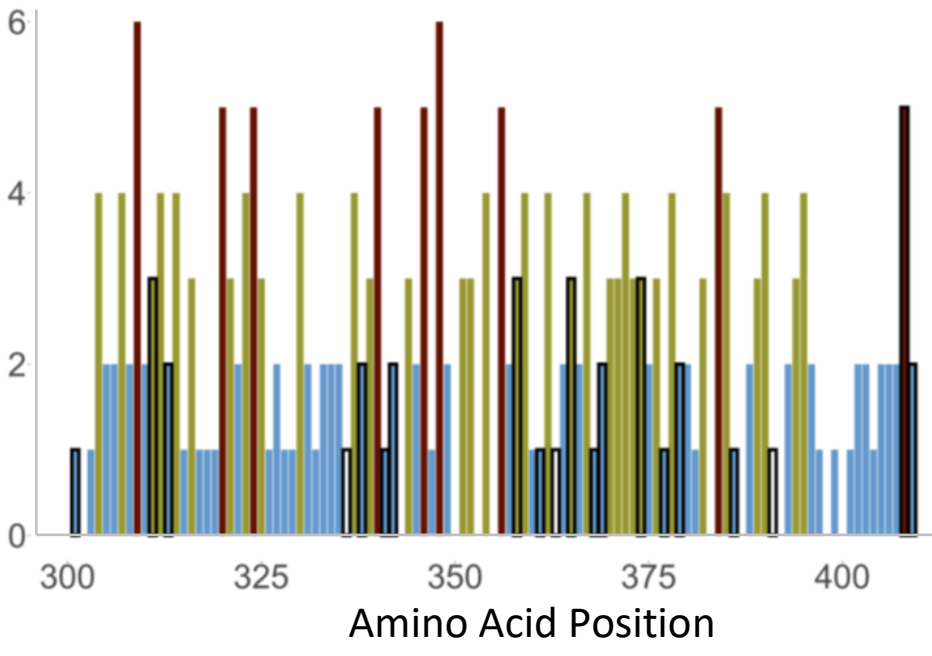

c

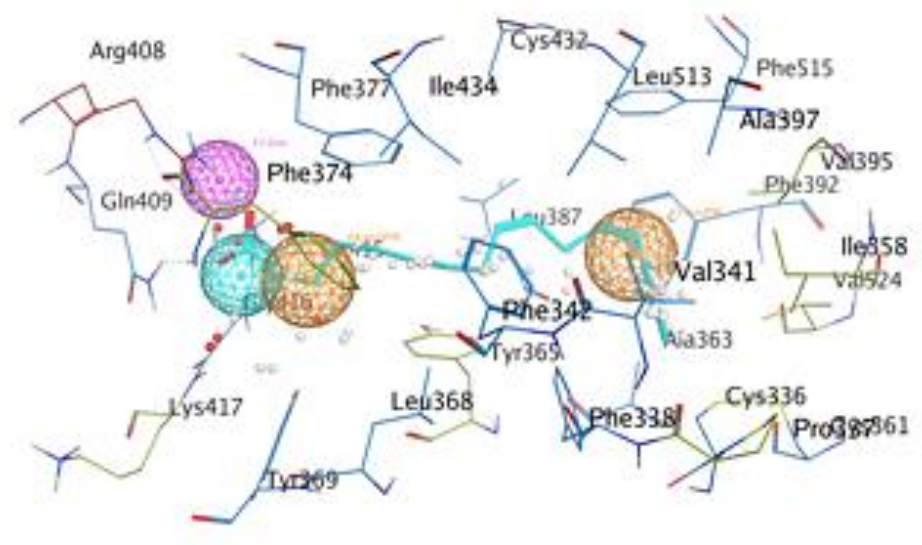


Figure 4. A relatively invariant ('hot') region in the S protein with no known function, identified by analyzing 441,168 individual virus sequences. a) The number of variants per position across the less-variable, 'hot' region with un-assigned function. The red star identifies the proposed 'latch', Q564 residue. b) The hot region identified in the 3-D structure of S (open conformation). c) Invariant 'hot' region in $S$ protein with un-assigned function depicted in both the open (left) and closed (right) conformations. Dark blue denotes invariant amino acids and light blue denotes positions with 1-2 observed variants. This region becomes exposed after $\mathrm{S}$ protein gets activated by proteases. d) Number of variants in hydrophobic patch with unassigned function. Positions outlined in black are part of the 'hotspot'. e) Some residues in the hotspot (shown in d) are part of the largest hydrophobic patch (green, red ellipsoid) of S protein. Positive patches are highlighted in red. Negative patches are highlighted in blue. f) Sequence identity between SARS-CoV-2 \& SARS-CoV (81\% identical), and SARS-CoV-2 \& MERS-CoV (15\%) in the 'hotspot'. Dark blue denotes identical amino acid residues. Numbering corresponds to SARS-CoV-2.
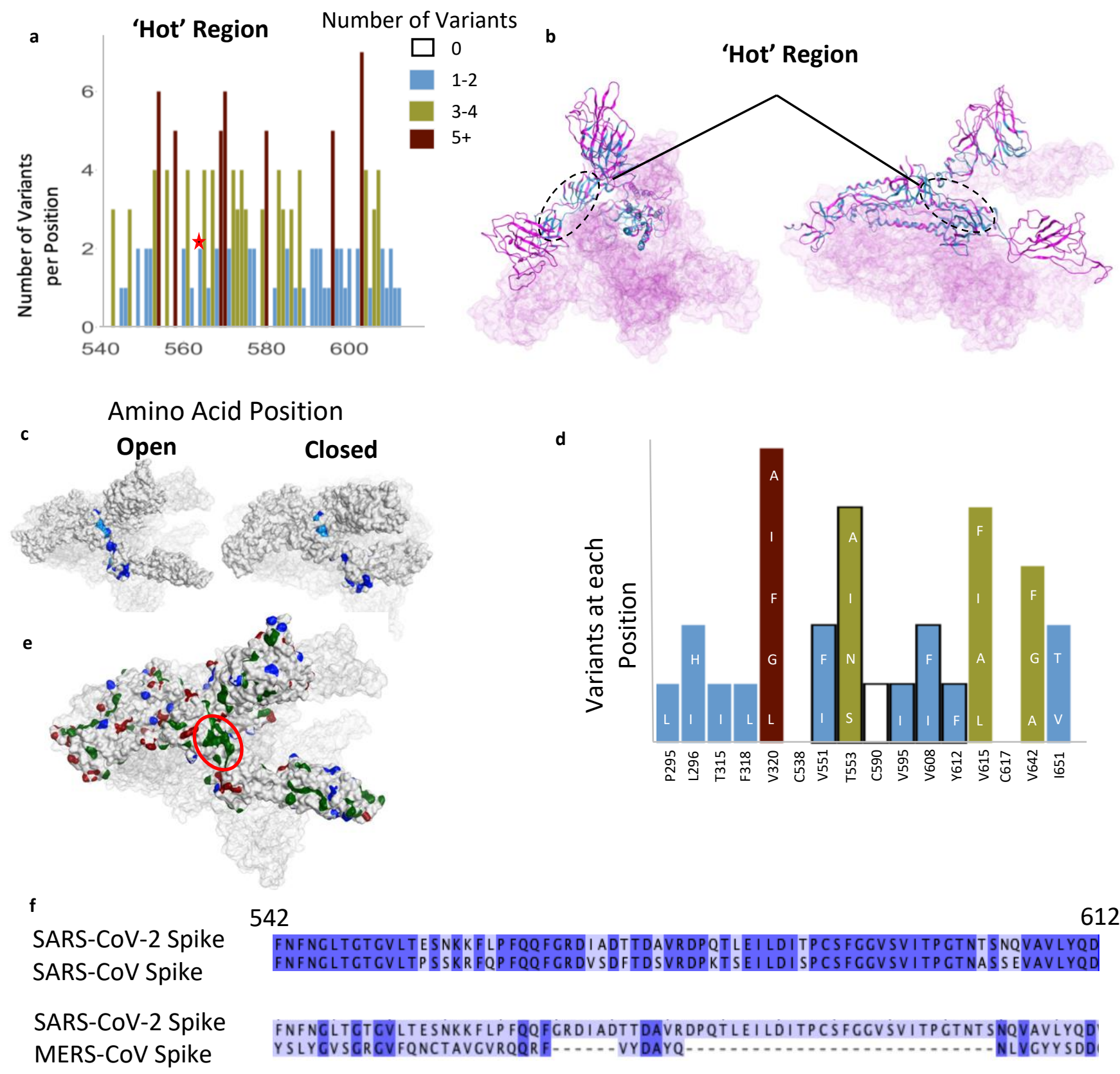

542 


\section{Natural variants in SARS-CoV-2 S protein pinpoint structural and functional hotspots: implications for prophylaxis and therapeutic strategies}

Suman Pokhrel ${ }^{1 \dagger}$, Benjamin R. Kraemer ${ }^{1 \dagger}$, Scott Burkholz ${ }^{2}$ and Daria Mochly-Rosen ${ }^{1 *}$

${ }^{1}$ Department of Chemical and Systems Biology, Stanford University School of Medicine, Stanford, CA

${ }^{2}$ Flow Pharma, Inc., Pleasant Hill, CA

†These authors contributed equally to the study

*Address correspondence to Daria Mochly-Rosen

Department of Chemical \& Systems Biology, School of Medicine, Stanford University, CA, USA

Email: mochly@ stanford.edu Tel: 650-724-8098 
Supplementary figure 1. Variant and invariant regions in SARS-CoV-2 protein. a) SARS-CoV2 Spike (S) protein trimer showing the receptor-binding domain (RBD) in the open conformation (red; PDB ID: 7A98) and the closed conformation (green; PDB ID: 6ZB5). Viewed from the top. b \& c) Regions in the sequence (1-636: b; 637-1273:c) of the S protein are color-coded according to function. d) New potential glycosylation sites (cyan) on $\mathrm{S}$ protein in circulating variants. e) Potential ligand binding site in the hotspot (aa 541-612).

$\mathbf{a}$

b Spike Protein
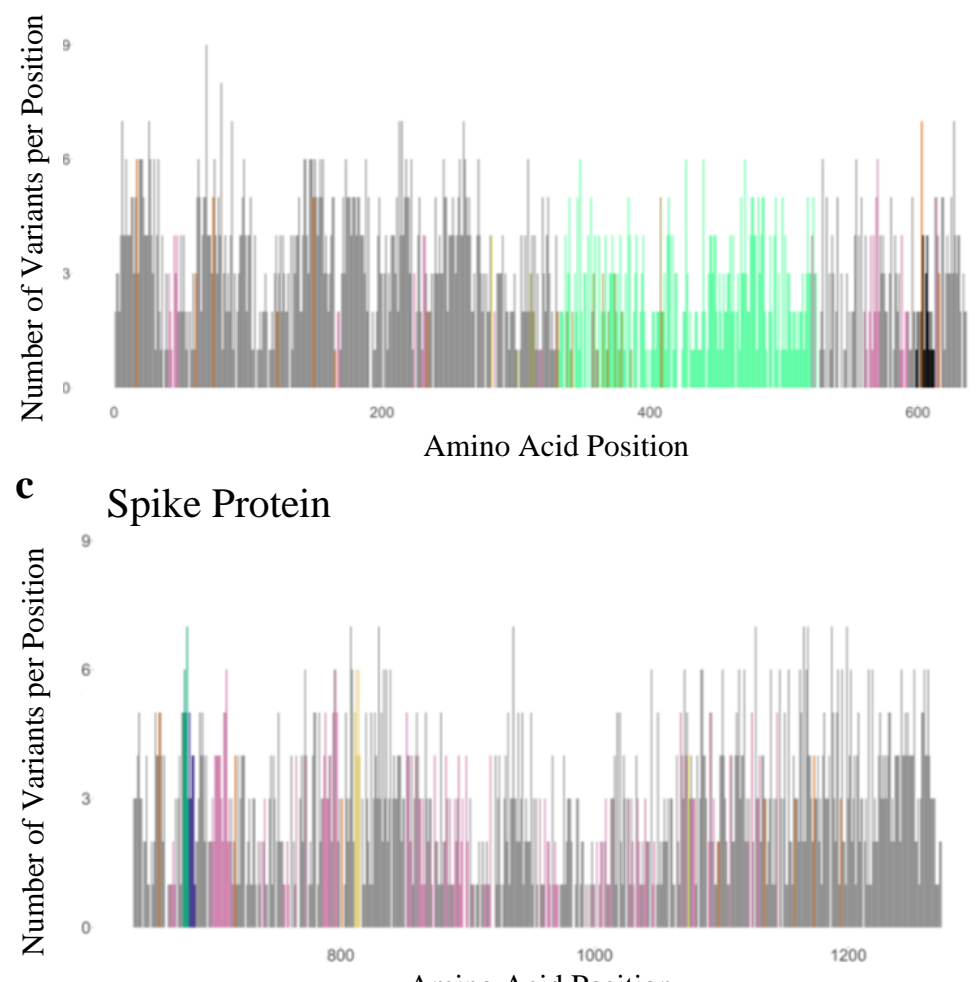

d

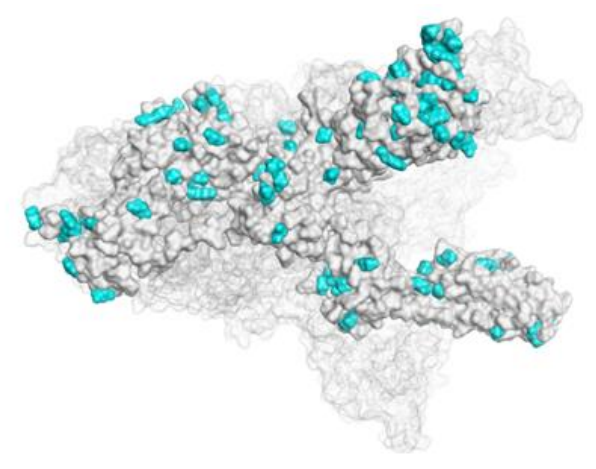

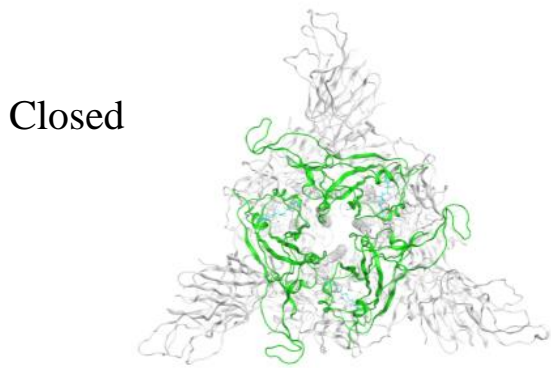

Functional Regions

Glycosylation Site Glvcosvlation Site/Monomer-Monomer Interaction LA Binding Monomer-Monomer Interaction RBD

Unidentified Fxn 'Hotspot'

Furin Site 2

NRP1 Binding

NRP1 Binding/Furin Site 1

NA

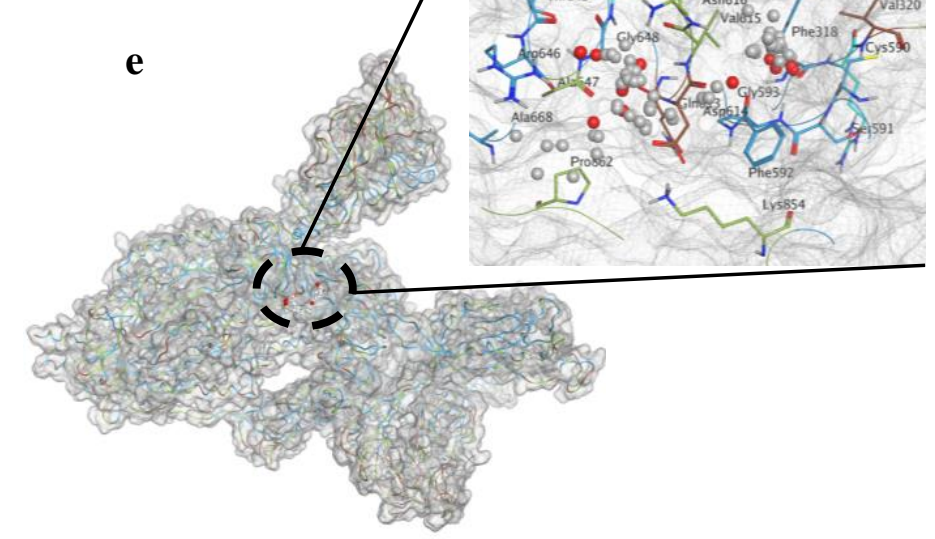

